\title{
Arabidopsis thaliana: a novel biocatalyst for asymmetric reductions
}

\author{
Satomi Takeda*,1, Yuka Ogata ${ }^{1}$, Hideo Kojima², Akiko Okada², Yosuke Uranishi², Kaoru \\ Nakamura ${ }^{3}$ \\ ${ }^{1}$ Department of Biological Science, Graduate School of Science, Osaka Prefecture University, Sakai, Osaka 599- \\ 8531, Japan; ${ }^{2}$ Department of Chemistry, Graduate School of Science, Osaka Prefecture University, Sakai, Osaka 599- \\ 8531, Japan; ${ }^{3}$ Graduate School of Human Development and Environment 'Science Shop', Kobe University, Kobe, \\ Hyogo 657-8501, Japan \\ *E-mail: takeda@b.s.osakafu-u.ac.jpＴel: +81-072-254-9186Ｆax: +81-072-254-9163
}

Received March 8, 2010; accepted September 27, 2010 (Edited by J. Yamaguchi)

\begin{abstract}
We used Arabidopsis thaliana seedlings as biocatalysts for the reduction of ketones, and investigated the factors affecting the chemical yield and enantioselectivity of the reactions. One- to four-week-old Arabidopsis seedlings were incubated for $24 \mathrm{~h}$ in reaction mixture containing either aromatic or aliphatic ketone as a substrate. After the reaction, the ketones and corresponding alcohols were extracted and quantified. The results indicated that Arabidopsis seedlings can be used as a biocatalyst for asymmetric reduction of ketones such as trifluoroacetophenone, $t$-butyl acetoacetate, methyl benzoylformate, and 2-(trifluoroacetyl)thiophene. The highest chemical yields were observed in seedlings pre-incubated under light conditions and in leaves, suggesting that asymmetric ketone reduction might be related to photosynthesis. In contrast, the age and size of seedlings did not have a significant effect on chemical yield or enantioselectivity. The findings suggest that Arabidopsis, which is widely used as a model plant system, presents a new opportunity for biotransformation.
\end{abstract}

Key words: Arabidopsis thaliana, asymmetric reduction, biotransformation, photosynthesis.

Many kinds of biocatalyst have been successfully used in biotransformation research. Optically active compounds are useful intermediates for pharmaceuticals, agrochemicals and liquid crystals. The necessity for stereoselective synthesis of optically active compounds with biological activities is important because only one isomer among the many optically active compounds available has a specific biological activity (Gotor et al. 2008). Isolated enzymes, microbes such as yeast and fungi, and plant cell cultures have been used as biocatalysts of asymmetric reductions (Matsuda et al. 2009). Microbes and plant cell cultures are more efficient biocatalysts than isolated enzymes because living cells provide an environment that includes substrates, numerous enzymes, cofactors, and cofactor regeneration systems, which facilitate biocatalytic reactions. However, it is not easy to obtain and maintain these kinds of cell cultures. Thus, other types of biocatalyst, namely, baker's yeast and vegetables, have also been applied to organic synthesis because they are readily available and easy to manipulate (Matsuda et al. 2009). However, the results obtained from experiments using this kind of biocatalyst, which are not produced under constant conditions, remain difficult to reproduce. This problem could be solved through the use of germinated plants. We are currently studying the possibility of using seedlings and cultured palnt cells as biocatalysts for asymmetric reduction of ketones, and recently, reported biotransformation using germinated radish (Matsuo et al. 2008)

Arabidopsis thaliana is widely used as a model organism for plant science research including genetics and plant development (Coelho et al. 2007; Rensink and Buell 2004). Seeds of $A$. thaliana are available worldwide and can be conserved for many years when stored under optimal conditions. Compared with other plants, Arabidopsis has a small genome, which was in fact one of the first genomes to be sequenced (The Arabidopsis Genome Initiative 2000). In addition, knockout and overexpression Arabidopsis mutants have been successfully characterized (Hayashi et al. 1992; Ito et al. 2005). In spite of these merits, A. thaliana is mainly used for researching the genetics, and cell and molecular biology of flowering plants, with little direct significance to agriculture- and biotransformation-related research. In the present work, we show that $A$. thaliana

Abbreviations: $t$ BAA, $t$-butyl acetoacetate; ee, enantiomeric excess; TFA, trifluoroacetophenone

This article can be found at http://www.jspcmb.jp/

Published online February 10, 2011 
seedlings can be used as a biocatalyst for asymmetric reduction of ketones.

Wild-type A. thaliana (L.) Heynh. ecotype Columbia was used in all the experiments. Surface sterilized seeds were plated on Murashige and Skoog medium (Murashige-Skoog salt mixture, 2\% (w/v) sucrose, $3 \mu \mathrm{g}$ $\mathrm{ml}^{-1}$ thiamin-HCl, $5 \mu \mathrm{g} \mathrm{ml}^{-1}$ nicotinic acid, $0.5 \mu \mathrm{g} \mathrm{ml}^{-1}$ pyridoxine- $\mathrm{HCl}$, and $0.2 \%$ gelrite, $\mathrm{pH} 5.8$ ), incubated for 3 days at $4^{\circ} \mathrm{C}$ in the dark then, unless otherwise mentioned, grown in a growth chamber at $23^{\circ} \mathrm{C}$ under short-day conditions $(8 \mathrm{~h}$ light $/ 16 \mathrm{~h}$ dark cycle). Arabidopsis seedlings were grown for 3 weeks, harvested, weighed, and transferred to a plastic tube containing $2 \mathrm{ml}$ of water for use as biocatalysts for reduction of ketones. A total of $13 \mu \mathrm{l}$ of ketone solution $(10 \%(\mathrm{w} / \mathrm{v})$ in DMSO) was added to the tubes and the reaction mixture was stirred at $100 \mathrm{rpm}$ at $25^{\circ} \mathrm{C}$ for $24 \mathrm{~h}$ under continuous white light (HITACHI FL40SW, ca. 30 $\mu$ mol photon $\mathrm{m}^{-2} \mathrm{~s}^{-1}$ ). The molar concentrations of each substrate in the reaction media were as follows: ketone 1a, trifluoroacetophenone (TFA), $0.99 \mathrm{mM}$; ketone 1b, $t$ butyl acetoacetate $(t \mathrm{BAA}), 0.43 \mathrm{mM}$; ketone $1 \mathrm{c}$, methyl benzoylformate, $0.71 \mathrm{mM}$; ketone $\mathbf{1 d}, \quad 2$ (trifluoroacetyl)thiophene, $1.40 \mathrm{mM}$. Next, the reaction media were sampled and extracted with ether solution containing naphthalene as an internal standard for gas chromatography. The conditions for GC were as follows: column; Varian Chirasil-DEX-CB $(25 \mathrm{~m} \times 0.32 \mathrm{~mm})$, TFA (1a); temperature $100^{\circ} \mathrm{C}$; retention time ketone, $1.64 \mathrm{~min}$; the $(S)$-alcohol, $7.89 \mathrm{~min}$; $(R)$-alcohol, 8.28 min, $t$ BAA (1b); temperature $120^{\circ} \mathrm{C}$; retention time ketone, $3.94 \mathrm{~min}$; the $(S)$-alcohol, $8.00 \mathrm{~min}$; $(R)$-alcohol, $8.42 \mathrm{~min}$, methyl benzoylformate (1c); temperature $130^{\circ} \mathrm{C}$; retention time ketone, $5.94 \mathrm{~min}$; the $(S)$-alcohol, $11.02 \mathrm{~min} ; \quad(R)$-alcohol, $\quad 10.52 \mathrm{~min}, \quad 2-$ (trifluoroacetyl)thiophene (1d); temperature $155^{\circ} \mathrm{C}$; retention time ketone, $1.77 \mathrm{~min}$; the $(S)$-alcohol, 7.44 $\min$; $(R)$-alcohol, $7.91 \mathrm{~min}$. The asymmetric reduction reaction of ketones using Arabidopsis as a biocatalyst is illustrated in Figure 1. Chemical yields (alcohols/ $($ ketones + alcohols $)) \times 100$ and enantiomeric excess, ee
$(\mid(S)$-alcohol- $(R)$-alcohol $/((S)$-alcohol $+(R)$-alcohol $)) \times$ 100 were obtained from GC-analysis using an internal standard (naphthalene).

Arabidopsis seedlings were able to reduce TFA (1a) mainly to the corresponding $(R)$-alcohol, $(R)$-2a. $t$ BAA (1b) was also reduced to the corresponding $(S)$-alcohol, $(S)$-2b. Although these ketones gave $(R)$ - and $(S)$ alcohols, the absolute configurations were the same due to definition. The chemical yields and ee for the reduction of TFA and $t$ BAA are shown in Figure 2. Surprisingly, the chemical yields, normalized by $1 \mathrm{mg}$ of biocatalyst, did not change significantly when seedlings grown for 1 to 4 weeks were used as biocatalysts (Figure 2A, B). To investigate the effect of seedling size, we used seedlings that, in spite of having been grown for the same period (3 or 4 weeks), exhibited different sizes. As shown in Figure 2, no significant change in chemical yield was observed when seedlings of different size were used as biocatalysts. In the same way, the enantioselectivity of the reactions was independent of the age and size of the seedlings. The ee $(R)$ of TFA reduction was between $28-36 \%$ (Figure $2 \mathrm{~A}$ ) and the ee $(S)$ of $t$ BAA reduction was always over $99 \%$ (data not shown). From these results, we concluded that the age and size of Arabidopsis seedlings did not affect the chemical yield or ee of the asymmetric reduction reactions. In other words, Arabidopsis seedlings can be used as biocatalysts regardless of their age and size. In contrast, chemical yields of microbial transformation usually depend on the growth stage (Matsuda et al. 2008).

In order to evaluate the biocatalytic efficiency of different Arabidopsis organs, we performed the reduction reactions using leaves, petioles, and roots derived from seedlings as biocatalysts. Figure 3 shows that the weightnormalized chemical yields for both TFA and $t$ BAA were high when photosynthetic organs such as leaves or petioles were used. In contrast, the biocatalytic efficiency of roots was very low (Figure 3A, B). These results indicate that asymmetric reduction of ketones mainly occurs in photosynthetic organs.

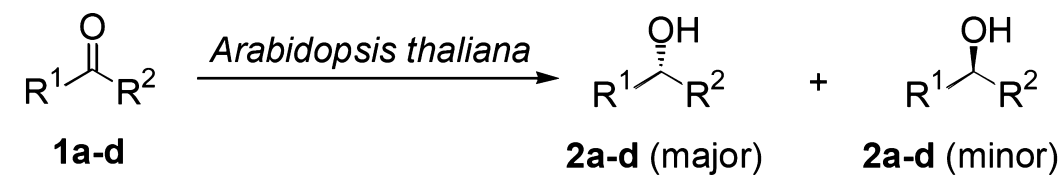

\begin{tabular}{lcc|c}
\multicolumn{1}{c|}{ Ketone } & $\mathrm{R}^{1}$ & $\mathrm{R}^{2}$ & Alcohol (major) \\
\hline 1a : trifluoroacetophenone (TFA) & $\mathrm{CF}_{3}$ & $\mathrm{Ph}$ & $(R)-\mathbf{2 a}$ \\
1b : $t$-butyl acetoacetate (tBAA) & $\mathrm{Me}$ & $\mathrm{CH}_{2} \mathrm{CO}_{2} \mathrm{Bu}^{\mathrm{t}}$ & $(S)-\mathbf{2 b}$ \\
1c : methyl benzoylformate & $\mathrm{CO}_{2} \mathrm{Me}$ & $\mathrm{Ph}$ & $(R)-\mathbf{2 c}$ \\
1d : 2-(trifluoroacetyl)thiophene & 2-thienyl & $\mathrm{CF}_{3}$ & $(R)-\mathbf{2 d}$
\end{tabular}

Figure 1. Asymmetric reduction of ketones 1a-d using Arabidopsis seedlings. 
Next, to determine the percentage of reaction products remaining in the plant, we extracted the reduction products from both plants and reaction media. About $85 \%$ of the TFA reduction products and $90 \%$ of the $t$ BAA reduction products were found in the reaction media (Figure 4). These results indicate that the reduction products can be easily collected at the end of the reaction.
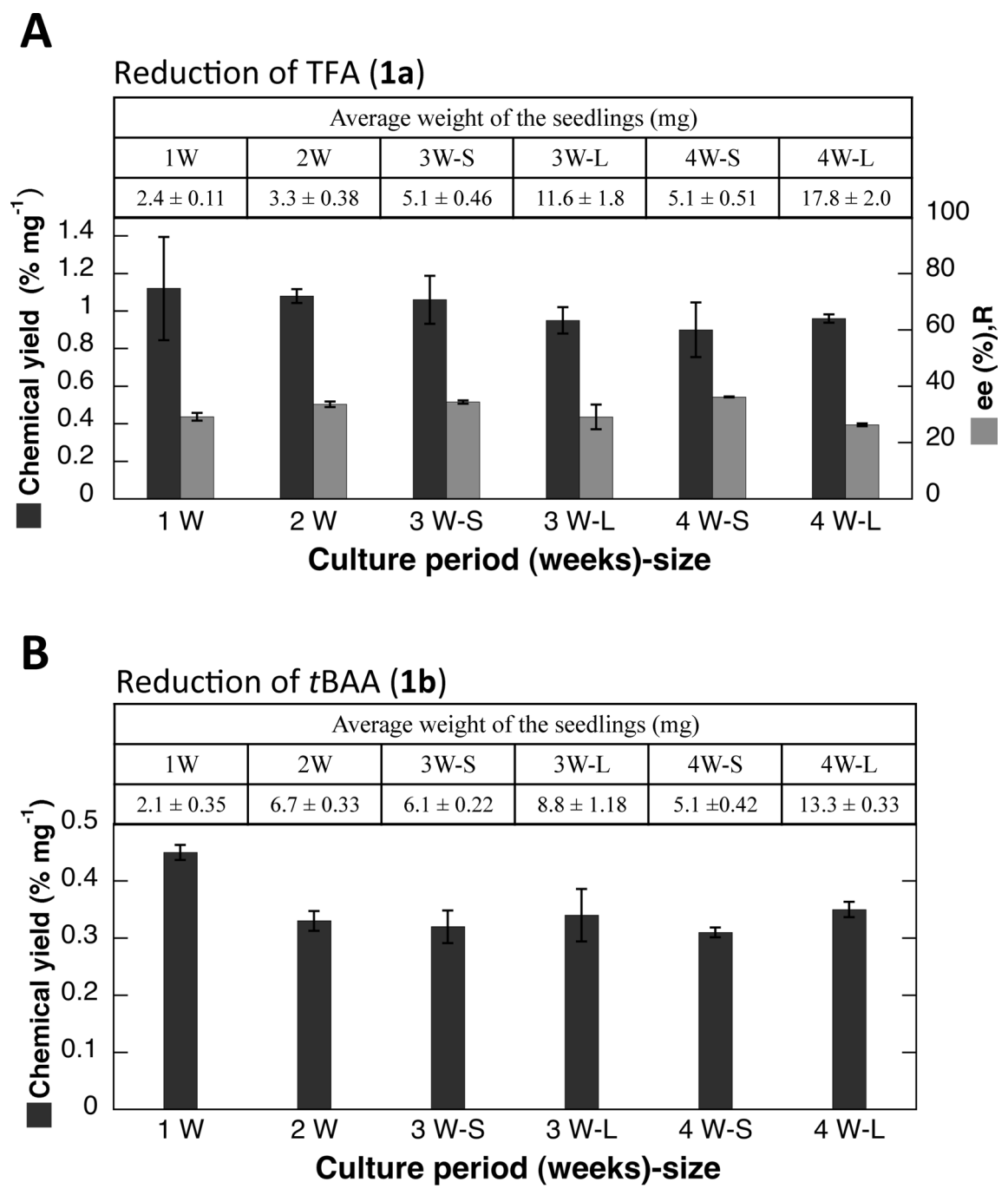

Figure 2. Effects of age and size of Arabidopsis seedlings on the reduction of trifluoroacetophenone (TFA, 1a) and $t$-butyl acetoacetate ( $t$ BAA, 1b). Seedlings were grown for 1 to 4 weeks $(1 \mathrm{~W}-4 \mathrm{~W})$. W-S and W-L represent seedlings that, despite having been grown for the same time, exhibited small and large sizes, respectively. The values presented are the averages and standard errors $(\mathrm{n}=3)$. (A) Chemical yield and ee $(R)$ of the TFA reduction reaction. (B) Chemical yield of the $t$ BBA reduction reaction. The ee $(S)$ of the $t$ BAA reduction was over $99 \%$ (not shown).
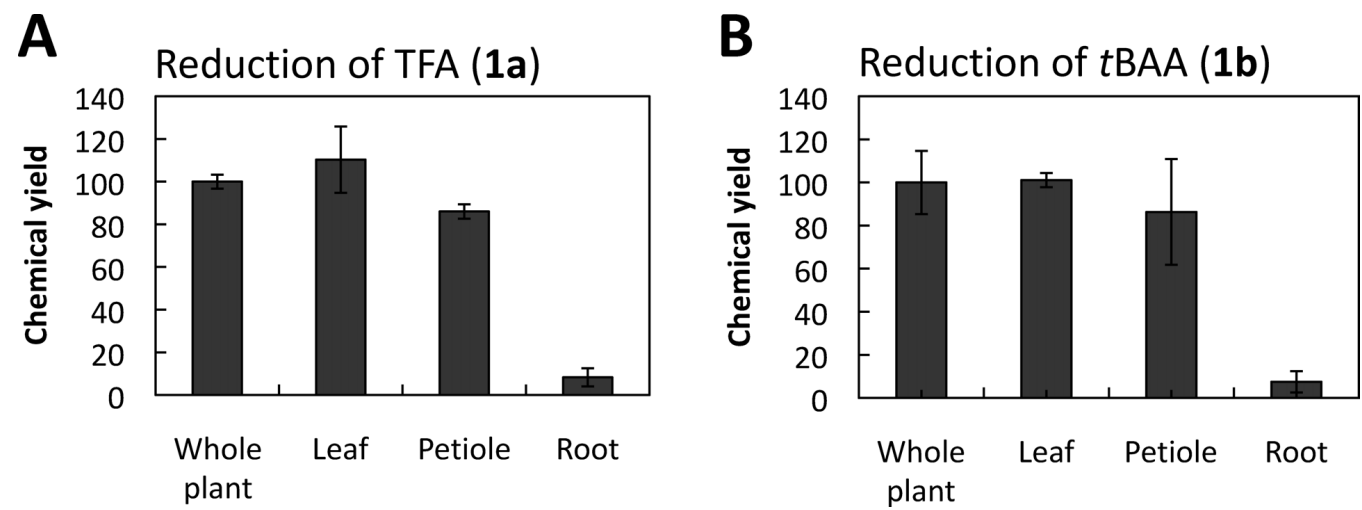

Figure 3. Chemical yield of the TFA (A) and $t$ BAA (B) reduction reactions using different Arabidopsis organs as biocatalysts. Chemical yield for each organ was expressed relative to the whole plant. The values presented are the averages and standard errors $(\mathrm{n}=3)$. 
Plant cell cultures can also be used as biocatalysts (Matsuda et al. 2009). Table 1 shows the chemical yields and ee when cultured Arabidopsis cells were used as biocatalysts. The cultured cells, which were maintained in LS medium (Linsmaier and Skoog 1965) supplemented with $10^{-5} \mathrm{M}$ naphthaleneacetic acid and $10^{-6} \mathrm{M}$ benzyl adenine under continuous light conditions, were diluted in fresh LS medium $(120 \mathrm{mg}$ $\mathrm{ml}^{-1}$ ), transferred to culture plates (Corning Incorporated, NY), and pre-cultured for 5 days under continuous light conditions. The substrate $(1.3 \mathrm{mg}$ of ketone dissolved in $13 \mu \mathrm{l}$ DMSO) was added to $2 \mathrm{ml}$ cell suspension and the reaction was carried out for $24 \mathrm{~h}$ in the light. The weight-normalized chemical yields using cultured cells as biocatalysts were low (Table 1), compared with those obtained from seedlings (Figure 2). Thus, the results indicate that the method using seedlings as biocatalyst is more efficient than that using cultured

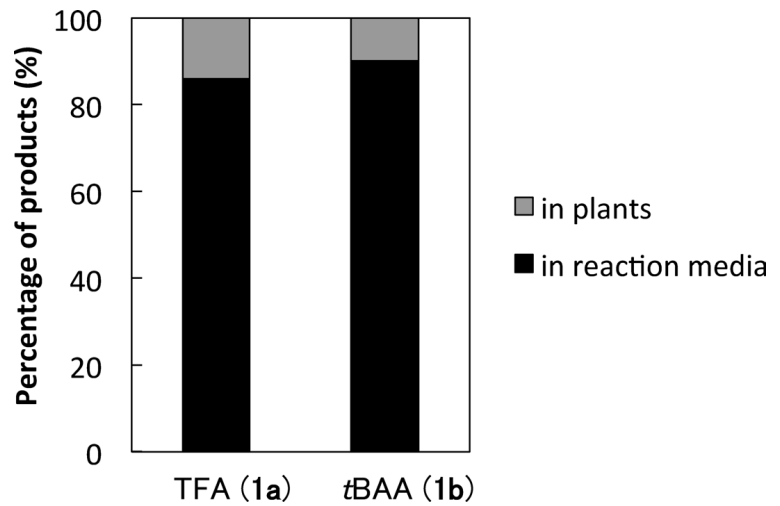

Figure 4. Location of the TFA (1a) and $t$ BAA (1b) reduction products. Key: in plants, the products remained in the plants; in reaction media, the products secreted to the reaction media.

Table 1. Chemical yield and ee of the Arabidopsis cultured cells mediated TFA (1a) and $t$ BAA (1) $)$ reduction products.

\begin{tabular}{ccc}
\hline substrate & Chemical yield $\left(\% \mathrm{mg}^{-1}\right)$ & ee $(\%)$ \\
\hline TFA & $0.261 \pm 0.008$ & $40 \pm 2$ \\
$t$ BAA & $0.225 \pm 0.012$ & $95 \pm 1$ \\
\hline
\end{tabular}

ee: enantiomeric excess
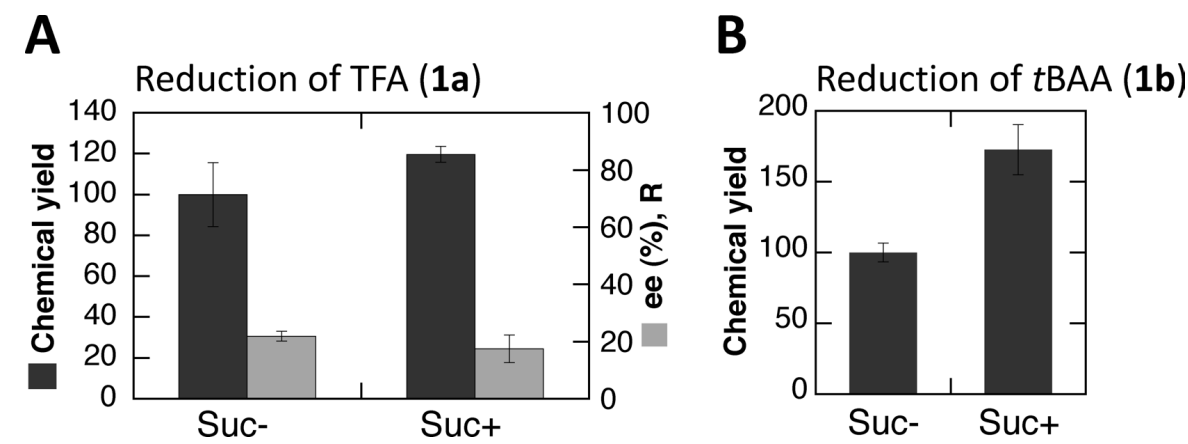

Figure 5. Effect of sucrose on the reduction of TFA (1a) and $t$ BAA (1b). Suc-, no sucrose in the reaction media; Suc,$+ 2 \%$ sucrose in the reaction media. Chemical yield for Suc + was expressed relative to Suc-. The values presented are the averages and standard errors $(n=3)$. (A) Chemical yield and ee $(R)$ of the TFA reduction reaction. (B) Chemical yield of the $t$ BBA reduction reaction. The ee $(S)$ of the $t$ BAA reduction was over $99 \%$ (not shown). 
A
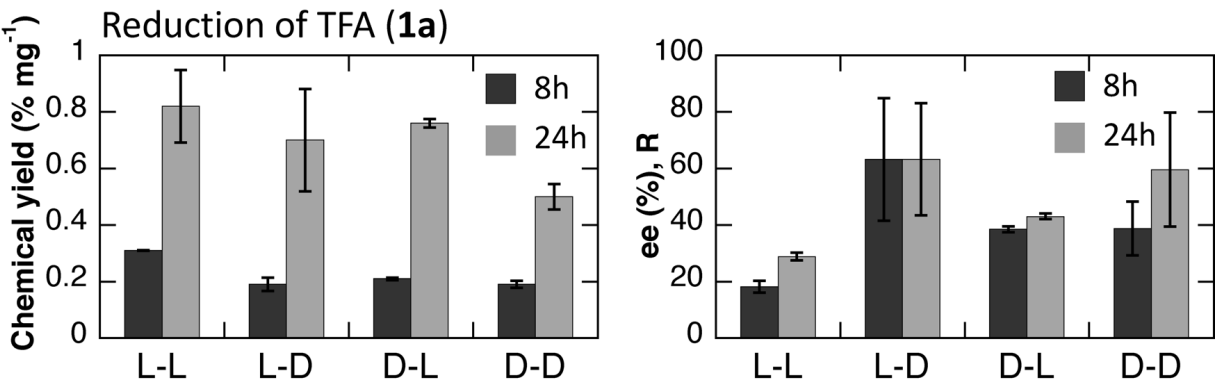

B

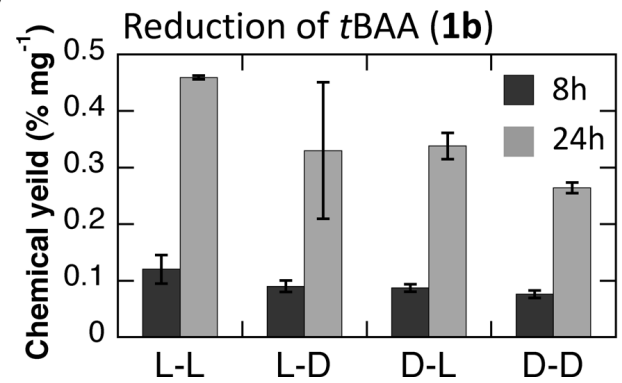

Figure 6. Effect of light on the reduction of TFA (1a) and $t$ BAA (1b). The seedlings were pre-cultured in the light and the reactions were performed under light $(\mathrm{L}-\mathrm{L})$ or dark $(\mathrm{L}-\mathrm{D})$ conditions. In addition, the seedlings were pre-cultured in the dark and the reactions were performed under light (D-L) or dark conditions (D-D). The values presented are the averages and standard errors $(\mathrm{n}=3)$. $(\mathrm{A}) \mathrm{Chemical}$ yield and ee $(R)$ of the TFA reduction reaction. (B) Chemical yield of the $t$ BAA reduction reaction. The ee $(S)$ of the $t$ BAA reduction was over $99 \%$ (not shown).

(D-D). Seedlings pre-cultured in the light ( $\mathrm{L}-\mathrm{L}$ and L-D) gave a higher chemical yield than those precultured in the dark (D-L and D-D), respectively. This may be explained by sufficient accumulation of reducing power during the light-pre-culture period, thus allowing efficient conversion of the ketone substrate into alcohol under dark conditions. The same phenomenon was observed in cultured tobacco cells (Kojima et al. 2009). In the case of enantioselectivity, the ee $(S)$ in the reaction of $t$ BAA using seedlings was extremely high regardless of the light condition. In contrast, enantioselectivity $(R)$ of the reduction of TFA in the light was lower than that in the dark (Figure 6A), suggesting that the $(S)$-alcohol producing enzyme might be activated in the light.

We also examined the asymmetric reduction reaction using other ketones as substrates. Arabidopsis seedlings catalyzed the reduction of methyl benzoylformate (1c) and 2-(trifluoroacetyl)thiophene (1d) to alcohols with moderate enantioselectivities (Figure 7).

The results presented in this work indicate that Arabidopsis seedlings can be used as biocatalysts for efficient reduction of ketones to alcohols, dependent on photosynthesis or photosynthetic products. In particular, when $t$ BAA was used as a substrate, optically active alcohol was obtained at a high chemical yield with excellent enantioselectivity. Since the chemical yields and enantioselectivities of the reactions did not significantly change with the age or size of the seedlings and since most of the reduction products were secreted to the reaction media, we believe that the use of

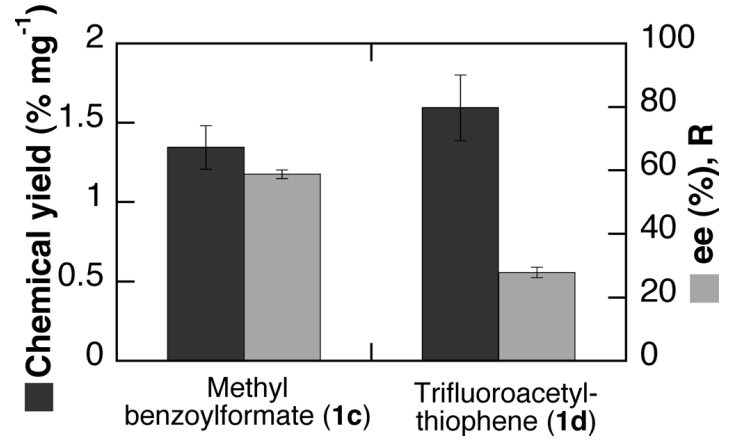

Figure 7. Reduction of methyl benzoylformate (1c) and 2(trifluoroacetyl)thiophene (1d) by Arabidopsis seedlings. The values presented are the averages and standard errors $(n=3)$.

Arabidopsis seedlings as biocatalysts will be a straightforward method of asymmetric reduction. Recently, Takemura et al. reported the characterization of a cyanobacterium knockout mutant defective in asymmetric reduction of ketones (Takemura et al. 2009). In cyanobacterium, these redox enzymes such as short chain alcohol dehydrogenase, aldo-keto reductase, and 3oxyacyl-(acyl-carrier protein) reductase are effective for the reduction of artificial ketones. We believe that similar enzymes may have contributed to the present reduction of ketones in Arabidopsis. We also believe that the characterization of Arabidopsis mutants exhibiting altered biocatalytic properties and cloning of the responsive genes will be an excellent tool for elucidation of the molecular mechanisms of asymmetric reduction 
reactions, thus helping to improve efficiency and selectivity.

\section{References}

Coelho S, Peters A, Charrier B, Roze D, Destombe C, Valero M, Cock J (2007) Complex life cycles of multicellular eukaryotes: new approaches based on the use of model organisms. Gene 406: $152-170$

Gotor V, Alfonsa I, Garcia-Urdiales E (2008) Asymmetric Organic Synthesis with Enzyme. Wiley-VCH, Weinheim

Hayashi H, Czaja I, Lubenow H, Schell J, Walden R (1992) Activation of a plant gene by T-DNA tagging: auxin-independent growth in vitro. Science 258: 1350-1353

The Arabidopsis Genome Initiative (2000) Analysis of the genome sequence of the flowering plant Arabidopsis thaliana. Nature 408: 796-815

Ito T, Motohashi R, Kuromori T, Noutoshi Y, Seki M, Kamiya A, Mizukado S, Sakurai T, Shinozaki K (2005) A Resource of 5,814 Dissociation Transposon-tagged and Sequence-indexed Lines of Arabidopsis Transposed from Start Loci on Chromosome 5. Plant Cell Physiol 46: 1149-1153

Kojima H, Okada A, Takeda S, Nakamura K (2009) Effect of carbon dioxide concentrations on asymmetric reduction of ketones with plant-cultured cells. Tetrahedron Lett 50: 7079-7081

Linsmaier EM, Skoog F (1965) Organic growth factor requirements of tobacco tissue cultures. Physiol Plant 18: $100-127$

Matsuda M, Otsuka Y, Jin S, Wasaki J, Watanabe J, Watanabe T, Osaki M (2008) Biotransformation of (+)-catechin into taxifolin by a two-step oxidation: Primary stage of $(+)$-catechin metabolism by a novel (+)-catechin-degrading bacteria, Burkholderia sp KTC-1, isolated from tropical peat. Biochem Biophys Res Commun 366: 414-419

Matsuda T, Yamanaka R, Nakamura K (2009) Recent progress in biocatalysis for asymmetric oxidation and reduction. Tetrahedron: Asymmetry 20: 513-557

Matsuo K, Kawabe S, Tokuda Y, Eguchi T, Yamanaka R, Nakamura K (2008) Asymmetric reduction of ketones with a germinated plant. Tetrahedron: Asymmetry 19: 157-159

Rensink W, Buell C (2004) Arabidopsis to rice. Applying knowledge from a weed to enhance our understanding of a crop species. Plant Physiol 135: 622-629

Takemura T, Akiyama K, Umeno N, Tamai Y, Ohta H, Nakamura K (2009) Asymmetric reduction of a ketone by knockout mutants of a cyanobacterium. J Mol Cat B-Enzymatic 60: 93-95 